\title{
Effective threat detection for surveillance
}

\author{
Judith Gelernter \\ School of Computer Science \\ Carnegie Mellon University \\ Pittsburgh, PA U.S.A. \\ gelern@cs.cmu.edu
}

\begin{abstract}
Surveillance video and protocols are only as effective as the people who are doing the monitoring. Threats might be overlooked because they are rare. Our experiment tested in the security domain what others have found in the medical domain: that repeating a rare event to make it less rare will make it more likely to be remembered, and hence, to be able to be reported. Results of our experiment supported the hypothesis that repeating rare events will make them more likely to be detected. We also looked at the relationship between threat detection performance and age, perceived level of attention, and recording procedure time devoted to each threat. Our specific findings concerning these factors led us to suggest actionable protocols to improve security officers' threat detection performance in video and live surveillance. Airport, border, urban, and other types of security screeners might benefit from these recommendations.
\end{abstract}

Keywords-surveillance, security camera, video, screening, monitoring, attention, inattentional blindness, Threat Image Projection (TIP) software, multi-tasking, airport screening

\section{INTRODUCTION}

A guard overlooking a threatening object or event might unintentionally cause a dangerous breech in security. But we should not blame the guard, because overlooking the unexpected is part of the human cognitive condition of "inattentional blindness". It is a by-product of selective attention that leads to tunnel vision, which is the limits of vision within a narrow channel. This research considers how to maximize the effectiveness of human monitoring.

\section{A. The data}

Many video streams can be united into a single monitor display to allow one person to oversee many locations simultaneously-this is the multi-panel, image-cycling video such as will show many areas inside or around a building. The state-of-the-art Domain Awareness System in New York City has an entire wall covered with monitors. ${ }^{1}$

\section{B. The human condition not to notice the unexpected}

Unexpected incidents might be seen but not remembered. This is because of the way the brain works, seeing what it

\footnotetext{
${ }^{1}$ NYPD, Microsoft create crime-fighting domain awareness tech system. http://www.huffingtonpost.com/2013/02/20/nypd-microsoft-domainawareness-crime-fighting-tech_n_2727506.html
}

expects to see only, and filtering the unexpected. ${ }^{2}$ The problem is aggravated when attention wanes over time (called "vigilance decrement"), or when people become distracted by noise or emotions.

\section{Effective threat detection within the human condition}

Overlooking rare events is a human pro-survival adaptation that appears on a neurological level [1]. Physiologically, it has been found that a person might see a rare event, but that event does not necessarily register in memory, and so it is as though it had not been seen [2]. Inattentional blindness has been more correctly called inattentional amnesia [3]. Our proposed solution is to help people remember what is rare by repeating the rare event. Note that this is also how people learn facts or new vocabulary words or sports' strategies-by repetition.

\section{STATE OF THE ART}

Covert tests by the U.S. Transportation Security Administration show that screeners continue to fall short in threat detection exercises [4]. This section describes a range of approaches being used currently to improve detection: (1) training personnel to recognize rare events, (2) computer vision methods to emphasize what is prominent in a video, (3) eye and vision studies for screeners, and (4) heightening memory of events via Threat Image Projection software.

\section{A. Personnel training}

Pre-employment vision tests for surveillance positions are sometimes required [5], but these constitute a fraction of what makes threat detection successful. Training screeners in what to look for is important. A recent study of Transportation Security Administration officers found some difference between naïve and experienced screeners [6]. Identification errors are in part to blame when naïve people miss more guns, knives and explosive devices than experienced screeners [7], [8]. Advances in threat types indicate that screener training must keep pace so that screeners know what to look for [9]. It was found that object identification is aided by practice looking at such objects in different rotations [10].

In inattentional blindness, people do not see events because the events are unexpected, not because they are not

\footnotetext{
${ }^{2}$ Selective attention is a biological adaptation and has been discovered in the placebo effect in which positive expectations control pain. Lidstone et al, (2010) Archives of General Psychiatry 67(8), 856-65.
} 
recognizable. A study of medical experts and novices in an inattentional blindness experiment found that experienced searchers did not perform very much better than those without experience [11]. Similarly, a study of results from threat image projection software found no correlation between amount of experience and detection performance [12].

\section{B. Computer vision}

Much research has been conducted on how to compress video or lessen noise in everyday activity video. Research has also been on how to draw attention to people or events that stand out, such as objects that have pre-defined features [13], or salient objects [14], or abnormalities [15]. Techniques are able to track the line of sight of people within the video so that those watching the scene can follow their gaze [16]. The video stream has been compressed so that it takes much less time to watch [17]. It could be altered to appear in three-dimensions [18]. The video stream could show just what moves [19], or the camera itself could move to encompass a wider field of view [20].

While bringing human activities into prominence will make it easier or faster to watch the video, most human activities are ordinary. Extraordinary events are still likely to be overlooked because they are unexpected.

\section{Vision studies}

Assumptions about the role of vision in improving threat detection performance have inspired research on the eye blink as an indicator of attention level [21]. Eyelids droop when people are drowsy, and fatigue can affect performance. However, inattentional blindness has been noted when people are not drowsy. That inattentional blindness has been detected in hearing as well as seeing [22], [23] is further reason to approach misses in visual search not as a vision problem but instead as problem of the brain and attention.

\section{Attention-related software}

One proposed solution to the attention problem is the Closed Loop Attention Management (CLAM) system which forces attention to an external stimulus [24]. But this works against the human tendency for attention to wane over longer periods of concentration, and so is unlikely to be effective long term.

Any sort of artificial-event software has the potential to be effective by working with the human tendency to notice what has been seen repeatedly. This principle underlies Threat Image Projection software. It projects artificial threat images onto actual suitcases, and has been used during working hours at airport checkpoints [25], and in other security domains [26]. Responses to the artificial events are used to assess visual search performance as well as heighten awareness of the potential for actual threats.

\section{OBJECTIVE AND HYPOTHESIS}

We describe below our experiment, its validity, and summarize findings (details are presented in [27]). While others have recommended protocols to increase surveillance monitoring effectiveness [28], our research lends scientific foundation to our recommendations.

\section{A. Hypotheses examined in experiment}

We examined the effect of increasing the frequency of a rare event to make it more likely to be seen, as has been found in the medical domain [29], [11]. We also examined the relationship of threat detection performance on factors such as reporting procedure time, age, perception of attention and location of events on screen.

\section{B. Generalizability of hypotheses}

Insight into the nature of overlooking the unexpected implies that a repeat-event software like the Threat Image Projection could be effective in lessening mistakes. Border security monitoring, urban or street monitoring, or searching for tumors in x-rays all could benefit from domain-specific repeat-event software because critical events are rare.

\section{Method AND NOVELTY}

\section{A. Our method}

We created three 2-hour surveillance videos alike in all but the number of low or high threat surprise events. Our 108 participants were assigned randomly to one of the three videos, in a between-group design. The primary task was to identify hat types and hat-wearer activities; the secondary task was to "call the police" (via mouse-click) a suspicious activity was seen. This resembles the standard two-task inattentional blindness experiment.

We trained participants in how to identify hats, low and high threat activities in order to minimize identification errors. The training lasted 30 minutes, in a session that preceded the two-hour experiment. We collected background information about each participant's age, logged each threat responded to and missed, and gathered exit data about attention level at the end of the study.

\section{B. Our novelty}

Ours is the only inattentional blindness experiment that we are aware of that is of extended duration. Most experiments last a few minutes [see bibliography in 27]. Unlike other inattentional blindness experiments, we do not mask the nature of the rare events and we give the participants numerous trials in which to spot events. The rare events (threats) differ from each other in kind, and in length, and in obviousness (whether one person or a group is involved). So while each participant has multiple tries (with the number of tries depending on what video watched) at the inattentional blindness task of threat identification, the threats themselves vary. 


\section{INSTRUMENT}

We consulted with surveillance software company WorldEyeCam on the design of authentic surveillance video display. $^{3}$ Typical of present-day small-scale surveillance video are four or more color panels showing different silent views of the surveillance space, with views changing every few minutes. In simulation of actual security footage, our video is multi-panel, in color, with no sound (Fig. 2). We recorded actors in 10 areas around one of the older buildings on our campus. We shot the footage in hallways without natural light so that we could stay for some time without changes in daylight making the passing of time obvious. That allowed us to combine all shoots later into a unified sequence.

Everyday activities in the videos include walking alone or in groups, chatting, trudging up sprinting down stairs, sitting in hallway chairs, and waiting for the elevator. Some wear hats. Our "skits" were tying shoelaces, holding a backpack, putting on lipstick, tearing an article out of newspaper, giving performance tickets to a friend, and taking off a jacket.

The surprise events in the videos are activities designated as suspicious by the National Terror Alert Center, ${ }^{4}$ and they are either classified as either low threat or high. We followed the Center's definitions in aiming for ecological validity, although numerous threats would not necessarily be seen in the space of a few hours. We created the videos so that there are fewer high threat activities than low in multi-threat videos $\mathrm{B}$ and $\mathrm{C}$.

Some of the low threat activities may be ordinary, but become sinister if used for malicious intent. High threat activities potentially result in immediate death or destruction. These appear in the videos as carrying a revealed weapon such as pistol, rifle, sword, or knife; carrying ammunition or bullets; planting an explosive device; or running away from a suitcase that might hold explosives.

\section{A. Experiment software}

We created Call \& Count software to accompany the video that would allow users to count hats or call the police upon spotting a threat. We designed the controls so that the keyboard input for hat counting would be easier than mouseclick input for threat calling since there were more hats than threats. We made it intuitive to differentiate between threat levels. One mouse click supposedly called the police to investigate a low threat, and a double click called the police to investigate a high threat.

An " $\mathrm{H}$ " cap covered each of four keyboard keys (R, C, U, $\mathrm{N}$ ) in a square to correspond spatially to the four panes of the video. Both threat-call and hat-count were location specific, so that the user was required to identify in which screen quadrant the event occurred. When a hat was spotted, the participant tapped the " $\mathrm{H}$ " key that corresponds to the panel where the hat was spotted.

\footnotetext{
${ }^{3}$ World Eye Cam, August 19, 2013 at http://www.worldeyecam.com/store/

${ }^{4}$ Low and high suspicious activities, February 12, 2013 at

http://www.nationalterroralert.com/suspicious-activity/
}

The control panel at the left of the video display shows the participant's response tally for hats and threat, and shows which quadrant the last call or count was input from (Fig. 2). Participants who noticed they have entered a hat or threat by

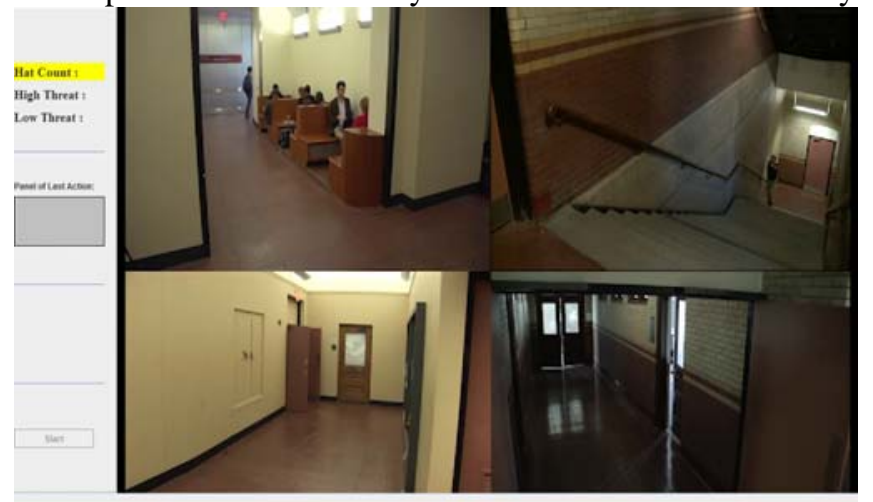

Figure 2. Shows a screenshot of the 4-panel demo video. Controls at left include a counter so that the participant can check his current hat and threat count, and the 4-quadrant rectangle that showed which quadrant the last call or count was input from.

mistake were urged to use the "Remove" bar (shown by label over the space bar on a standard computer keyboard) to undo the last action. The user had no ability to rewind the video since we are studying attention in real time.

\section{EXPERIMENT}

\section{A. Primary vs secondary task}

Increasing the amount of attention required by a primary task has been found to increase inattentional blindness for a secondary task [30], [31]. We increased the amount of absorption required for the hat-counting task by asking participants to remember throughout the experiment what the hats look like, where in the building the hat-wearer happens to be, whether the hat-wearer is alone or in a group, and whether the hat-wearer is walking, running, standing or sitting. Instructions before the experiment tell participants that they will be asked specific questions about hat-wearers at the end of their shift. Threat-identification task is presented as the secondary task by presenting it second in the instructions after the hat counting task. Also, it is shown as second in the layout of the control panel (Fig. 2). The threat-identification task required less concentration than the hat task, moreover, since there was nothing about each threat that needed to be remembered, as the locations of the hat wearers needed to be remembered.

\section{B. Secondary task and the experiment protocol}

We wanted to minimize errors due to threat identification as opposed to errors due to not seeing - the point of the experiment - so we set the experimental protocol to reinforce threat identification. The training in learning how to recognize low and high threat activities began by asking participants to read our Instruction sheet with definition of hats and threats and how to use the system controls. Then we encouraged them to ask questions about the instructions. We removed the 
instruction sheet while we asked them questions about the instructions in a game that we called 'Instructions Jeopardy', so that they would enjoy the preparation rather than feel that they were being drilled. Then we gave each participant a blank sheet of paper that asked them to list the four qualities of hat-wearers they should be looking for, the four types of low threats, and the four types of high threats. The principal investigator went from person to person discussing each participant's fill-in answers individually, before that participant was invited to start our short practice demo. The Instructions sheet with definitions of hats and high and low threats then were returned to participants for the demo and the course of the experiment.

The purpose of the under-two-minute demo was to provide familiarity with the display and practice responding to hats and threats by using the controls. Each participant had the option of re-running the demo to gain familiarity with the controls before starting the full experiment.

\section{Construct validity test: secondary task only}

We wanted to demonstrate that rare events are more easily spotted when event spotting is the only task, than when it is the secondary task. So, we created a separate set of instructions and a separate control panel for this "no-hat" experiment. Fifteen participants were assigned randomly to six groups: $\mathrm{A}, \mathrm{B}$ and $\mathrm{C}$ full experiment (with partial concentration to threat identification) or A, B and C no-hat experiment (with full concentration to threat identification), making 90 participants in all.

Results show that in the no-hat experiment people make fewer errors than in the full experiment (Fig. 3). That participants spot many more events accurately when they can devote full attention to threat detection demonstrates that the experimental concept is sound. Although we could prove this more certainty given a larger sample size, our findings demonstrate that the experiment is valid because people are less proficient at threat detection when they allot only partial concentration to the task.

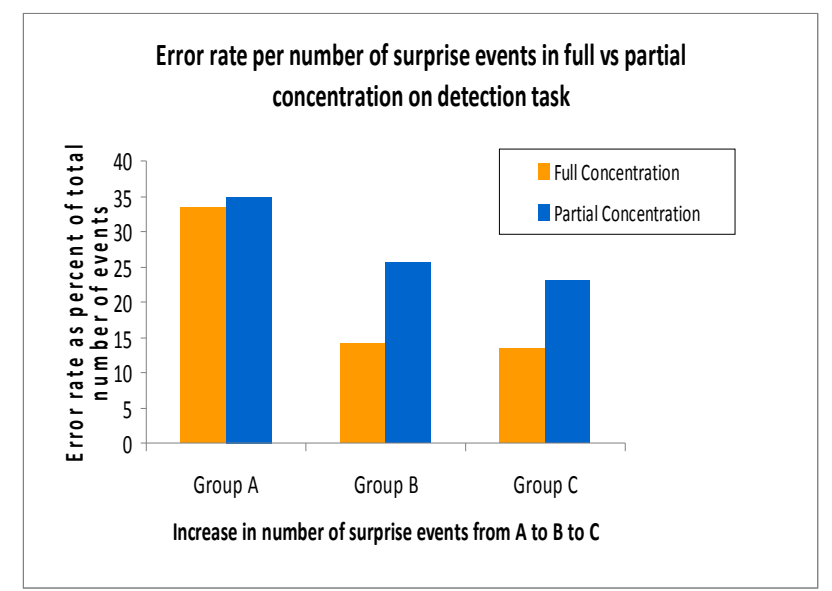

Figure 3. Concept validity experiment with $\mathrm{N}=90,15$ per group. Chart shows rare event detection in the full experiment (with hats) vs the partial experiment (threats-only)

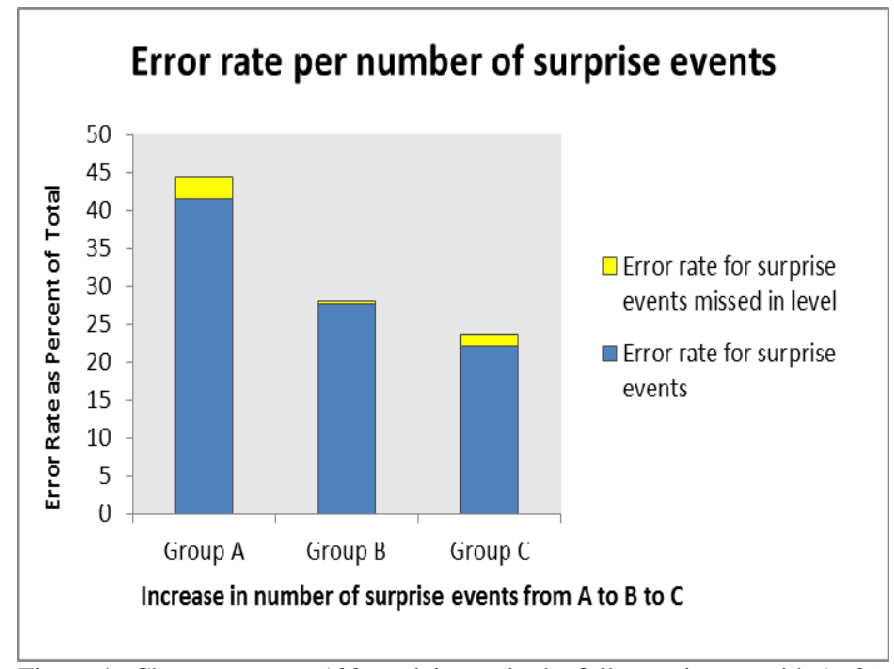

Figure 4. Chart represents 108 participants in the full experiment, with $\mathrm{A}=36$, $\mathrm{B}=36$ and $\mathrm{C}=36$ participants. Group A has 2 events, B has 9, and C has 25 surprise events. Events missed in level are low threats counted as high, and vice versa.

\section{RESUltS OF THE Full EXPERIMENT}

Some findings from our experiment as described in this section support recommendations in the following section to improve threat detection performance.

1. Responding to rare events makes it more likely that future actual events of a similar nature will be detected. We found a statistically significant difference between the control group and the group with the maximum number of events in terms of event reporting accuracy (Fig. 4). Software feedback from responses to simulated threats will allow the monitoring officer to see how well he is doing.

2. Multi-tasking is not efficient. Participants did less well on the primary hat-counting task as the number of surprise events to be detected increased. In other words, there is less available attention for one task as the attention requirements for a parallel task increase. Back-up screeners could lessen difficulties.

3. Self-perception of attention level does not match actual attention level. We found a gap between perceived and actual attention level during the first third of the study (when participants thought their concentration was keener than results showed), and during the last third of the study (when participants thought their concentration had dropped more than it had). This implies that self-assessment of attention level is unreliable.

4. Events in familiar locations are more easily detected. We found a significant correlation between the screen quadrant that people are accustomed to looking at, according to humancomputer interaction studies [32], and the quadrant where they made the fewest overlook mistakes. This implies that the 
effect of habit is strong not only in terms of what people are looking for, but also in terms of where they look.

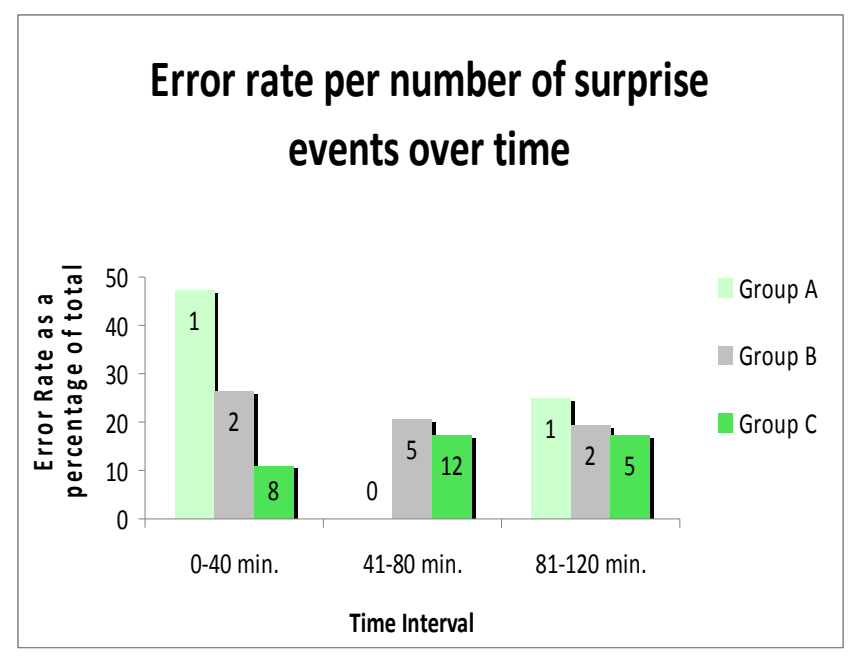

Figure 5. Chart represents 108 people in the full experiment, with $A=36$ $\mathrm{B}=36$ and $\mathrm{C}=36$ participants. It shows the time period of each group on the $x$ axis by the average error rate for surprise events on the $y$ axis. The labels in the data bars indicate the actual number of surprise events in each time segment of the video.

5. Age does not correlate with threat detection performance. We found no threat detection performance difference between participants in their teens and 20s and those in their senior years. Therefore, screening work that is on- or off-site should be open to older adults.

6. Threat detection performance lessens over a two-hour shift, but not significantly. The level of inattentional blindness did not decline significantly over the two hours of the experiment (Fig. 5). This might be explained by the fact that the focused thought becomes weaker over hours of sustained attention, and as the focus weakens, the level of inattentional blindness should weaken and the ability to notice unexpected improves.

7. Does thought time improve performance? We did not find that those who devoted more thought time to each surprise event made fewer overlook mistakes. But others have shown in practice that searches conducted more slowly are more likely to be effective [33]. So it is possible that our experimental protocol biased results.

\section{RECOMMENDATIONS TO IMPROVE THREAT DETECTION PERFORMANCE}

This section contains actionable protocols regarding human surveillance that should lessen the number of misses in visual searches by security staff. Recommendations should be tested in small scale to adjust for optimum performance prior to widespread adoption.

\section{A. Artificial events interspersed with actual objects or video}

1) Nature of the event
Repetition of a rare event should improve detection of an event similar in nature (with the theory explained in section I, and experimental results provided in section VII). The artificial rare event should resemble the actual in type and in duration.

Artificial event response systems can be made by overlaying clips of rare events over real-time video. The actual video under the clip could be routed to another surveillance officer, as described in the Back-up screening section below. The artificial event response systems require a method for the user to respond to events, a mechanism to record whether each artificial event was detected, and immediate or tallied feedback to let the user know whether he has found what he should.

It has been found that even when Threat Image Projection software is used by airport screeners, the screeners' covert tests with actual weapons may fail [33]. For this, we suggest interspersing threats with non-threats. The use of foils will force screeners to pay closer attention to projected images. Our main experiment suggested that the performance improvement (fewer visual mistakes) decreases as more and more artificial events are added. This should help optimize artificial event systems.

2) Incorporate a wider visual area into the artificial event protocol to help ensure that threats in this area are noticed

We found in experiment that people make fewer mistakes in parts of the screen where they are accustomed to looking. Hence, with artificial events appearing on screen areas that people are unaccustomed to examining-and feedback when people miss events in those areas-the visual range of screeners can be widened. Similarly, objects which people carried on their bodies in covert tests were discovered less often than those in luggage, where security officials have had threatimage projection training [33]. Covert test with weapons on the person as well as in luggage should be routine.

\section{B. Covert tests}

Covert tests are real-life simulations. They are conducted by officials who break rules in order to evaluate whether security is finding rule-breakers. Simulations staged or overlaid in real-time monitoring, if frequent enough, could help improve threat detection performance. Both types should serve to lessen mistakes due to overlooking rare events.

\section{1) Frequency}

Covert tests staged at irregular times of the day and at irregular intervals throughout the year will serve as practice to improve performance. Covert tests should be used in addition to simulations to lessen visual mistakes. The interval should not be too long between tests, since it has been found that personal effort level drops following an evaluation if the agent views the time until the next evaluation as long [34].

\section{2) Cost-saving team-work}

Staff could rotate in performing covert tests for each other. Because their faces would be familiar, many of their attempts 
should be innocent and only occasionally should they feign suspicious behavior or carry threatening objects. These covert tests could be organized by the workers themselves rather than delivered from outside. This reinforces a leadership model proposed especially for airport security [35].

\section{Back-up screening}

Off-site screening for redundancy is under used. An offsite employee would examine the same screen as an on-site person, and if responding to a threat, could send a warning to the on-site person. In high-traffic areas such as airport checkpoints, off-site guards could double the on-site staff for higher reliability. At other times, off-site screeners would allow the on-site people to have breaks between shifts, which, in turn, would allow them to be more attentive when they are on shift.

\section{Personnel selection}

Who are good candidates for guards and back-up guards? We mentioned above that overlooking rare events is a human characteristic, and does not seem to correlate highly with experience or age. Those who retire early, or are housebound, would make fine off-site screeners, as age has been found to be irrelevant in threat detection performance.

\section{E. Motivation}

Positive motivation has been found "to elicit desired behavioral responses and performance levels” [34]. Individual evaluation might lead to unwanted competitiveness within a site, when really what is wanted is full staff cooperation. So the most effective positive motivation might be to evaluate the team rather than the group on what they find rather than what they miss. Reward might take the form of team recognition, time off, or financial compensation.

\section{FURTHER STUDY}

The basis for lessening inattentional blindness toward rare events to make them more familiar, and therefore more able to be noticed, should be tested to determine the optimum frequency of artificial events. We do know with certainty that these artificial events must be at irregular intervals so that they retain their effectiveness.

\section{CONCLUSION}

Surveillance video is only as effective as the person who monitors it. We have produced experimental evidence that the number of overlook errors for rare events is less when rare events are peppered into the regular search routine. This will help the screener remember what was seen. The principle could be applied to the creation of systems in any surveillance situation.

The idea is to overlay simulated rare events on real-time video. Screeners would have the option of responding to those events, and the benefit of seeing feedback from their responses. Repeating rare events is the idea behind Threat Image Projection (TIP) software already in use in some airport security checkpoints. We have suggested ways to make this and similar artificial threat software more effective by the addition of more covert testing, off-site backup screening, and positive motivation to the screener team.

\section{ACKNOWLEDGMENTS}

Gang Wu and Sally Gao developed the video software with some help from Apoorvi Jain. Data analysis was conducted by Apoorvi Jain, with advanced statistics from Daniel Shriner. Kevin Gilbert edited the manuscript. Thanks to Elmar Schmeisser, whose invitation to submit a proposal helped made this research possible, and to Jaime Carbonell, who had the idea of using two levels of threats, and who supported the research when it was threatened financially. The work of the actors who made the video, and videographers Jim Kapustik and Rich Noel from the Post Modern studio who recorded and edited the footage, is also appreciated. Our work would be meaningless without data from the people who spent hours participating in our experiment.

Major support for the research comes from ARL/ARO award 60355-LS-II. A Berkman Faculty Development Grant to cover creation of the videos was awarded by Carnegie Mellon University.

\section{REFERENCES}

[1] Cisek, P., Puskas, G.A., El-Murr, S. (2009). Decisions in changing conditions: the urgency-gating model, Journal of Neuroscience, 29 (37), 11560-11571.

[2] Castel, A., Vendetti, M., Holyoad, K.J. (2012). Fire drill: Inattentional blindness and amnesia for the location of fire extinguishers. Attention, Perception and Psychophysics 74 (7), 1391-1396.

[3] Wolfe, J. (1999). Inattentional amnesia. In V. Coltheart (Ed.), Fleeting Memories (pp.71-94). Cambridge, MA: MIT Press.http://search.bwh.harvard.edu/pdf/fleeting_memories\%20html.pdf

[4] Hallinan, J. (2009). Why we make mistakes: How we look without seeing, for things in seconds, and are all pretty sure we are way above average. New York: DoubleDay Publishing.

[5] Bolfing, A. and Schwaninger, A. (2009). Selection and pre-employment assessment in aviation security x-ray screening. $43^{\text {rd }}$ Annual Carnahan Conference on Security Technology 5-8 October 2009, Zurich, Switzerland, ICCST, 5-12.

[6] Biggs, A.T., Cain, M.S., Clark, K., Darling, E.F., Mitroff, S.R. (2013). Assessing visual search performance differences between Transportation Security Administration Officers and non-professional visual searchers. Visual Cognition 21(3), 330-352.

[7] Liu, X. and Gale, A. (2011). Air passengers' luggage screening: What is the difference between naïve people and airport screeners? Proceedings $9^{\text {th }}$ International Conference on Engineering psychology and cognitive ergonomics EPCE 2011, 9-14 July, 2011, Orlando, Florida, 424-31.

[8] Schwaninger, A., Hardmeier, D., Hofer, F. (2005). Aviation security screeners: visual abilities \& visual knowledge measurement. IEEE Aerospace and electronic systems magazine 20(6), 29-35.

[9] Neiderman, E.C. (2000). Development and validation of a test for X-ray screener readiness. International Carnahan Conference on Security Technology, 23-25 October, 2000, Ottawa, Ontario, Canada, 192-196. 
[10] Michel, S., Koller, S.M., de Ruiter, J.C., Moerland, R., Hogervost, M., Schwaninger, A. (2007). Computer-based training increases efficiency in $\mathrm{x}$-ray image interpretation by aviation security screeners. Proceedings $41^{\text {st }}$ annual IEEE international Carnahan Conference on Security Technology, 8-11 October, Ottawa, Ontario, Canada, ICCST, 201-206.

[11] Evans, K.K., Tambouret, R.H., Evered, A., Wilbur, D.C., and Wolfe, J.M (2011). Prevalence of abnormalities influences cytologists' error rates in screening for cervical cancer. Archives of Pathology and Laboratory Medicine 135 (12), 1557-60.

[12] Schwaninger, A., Hofer, F., Wetter, O.E. (2007). Adaptive computerbased training increases on the job performance of x-ray screeners. $41^{\text {st }}$ Annual IEEE Carnahan Conference on Security Technology, 8-11 October 2007, ICCST, 117-124.

[13] Fernandez-Caballero, A., Lopez, M.T., Fernandez,M.A., Mira, J., Delgado, A.E. (2006). Visual surveillance by dynamic visual attention method. Pattern Recognition 39(11), 2194-2211.

[14] Hsieh, M-D., Chen, S-B, Cai, Y-S, Chen, Y-F., Chiang, J.Y. (2010). Immersive surveillance for total situational awareness. 2010 International Computer Symposium (ICS 2010), 16-18 December, Tainan, Taiwan, 300-305.

[15] Zhi, Z., Yangsheng, X., Weiren, S., Weizhong, Y., Keung, L.K. (2007). Crowd abnormality surveillance. Chinese journal of scientific instrument $<$ sic $>28(4), 614-20$.

[16] Leykin, A., Hammoud, R. (2008). Real-time estimation of human attention field in LWIR and color surveillance videos. 2008 IEEE Computer Society Conference on Computer Vision and Pattern Recognition Workshops (CVPR Workshops), 23-28 June, 2008, Anchorage, AK, USA, 1-6.

[17] Peleg, S. (2013). BriefCam. http://www.pbs.org/wgbh/nova/tech/surveillance-video.html

[18] Barrientos, J. M. (2000). Screener threat detection performance with three dimensional X-ray imaging. Proceedings IEEE $34^{\text {th }}$ Annual 2000 International Carnahan Conference on Security Technology, 23-25 October, 2000, Ottawa, Ontario, Canada, ICCST, 178-191

[19] Szpak, Z.L. and Tapamo, J.R. (2010). Maritime surveillance: Tracking ships inside a dynamic background using a fast level-set. Expert systems with applications 38(6), 6669-80.

[20] Davis, J.W., Morison, A.M., Woods, D.D. (2007) An adaptive focus-ofattention model for video surveillance and monitoring. Machine Vision and Applications 18(1), 41-64.

[21] Vural, U., Akgul, Y.S. (2011). Operator attention based video surveillance. 2011 IEEE International Conference on Computer Vision Workshops, 6-13 November 2011, Barcelona, Spain, 1955-1962.

[22] Sinnett, S., Costa, A. Soto-Faraco, S. (2006). Manipulating inattentional blindness within and across sensory modalities. Quarterly Journal of Experimental Psychology (Colchester) 59(8), 1425-1442.

[23] Wayand, J.F., Levin, D.T., Varakin, D.A. (2005). Inattentional blindness for a noxious multimodal stimulus. American Journal of Psychology 118(3), 339-352.

[24] St. John, M. and Risser M.R. (2008). Closed-loop attention management using augmented cognition to sustain vigilance. Proceedings of the $26^{\text {th }}$ Army Science Conference, Washington, D.C. [7 p.].

[25] Steiner-Koller, S.M., Bolfing, A., Schwaninger, A. (2009). Assessment of X-ray image interpretation competency of aviation security screeners. 2009 IEEE $43^{\text {rd }}$ International Carnahan Conference on Security Technology ICCST 2009, 5-8 October, Zurich, Switzerland, 20-27.

[26] Neil, D. Thomas, N., Baker, B. (2007). Threat image projection in CCTV. International Carnahan Conference on Security Technology, 811 October, 2007, Ottawa, Ontario, Canada, ICCST, 272 - 280.

[27] Gelernter, J. (2013). Surprise event simulations can lessen mistakes caused by inattentional blindness. Technical unpublished report. School of Computer Science, Carnegie Mellon University, April 2013.

[28] Cutler, V. and Paddock, S. (2009). Use of threat image projection (TIP) to enhance security performance. 43 $3^{\text {rd }}$ Annual 2009 International Carnahan Conference on Security Technology, 5-8 October, Zurich, Switzerland, ICCST, 46-51.
[29] Evans, K., Cohen, M. A., Tambouret, R., Horowitz, T. Kreindel, E. and Wolfe, J. M. (2011). Does visual expertise improve visual recognition memory? Attention, Perception and Psychophysics 73(1), 30-35.

[30] Macdonald, J.S.P. and Lavie, N. (2008). Load induced blindness. Journal of Experimental Psychology: Human Perception and Performance 34(5) 1078-1091.

[31] Perez-Moreno, E., Conchillo, A. and Recarte, M.A. (2011). The role of mental load in inattentional blindness. Psicologica 32, 255-278.

[32] Nielson, J. and Pernice, K. (2010). Eyetracking web usability. Berkeley, Calif.: Nielson Norman Group.

[33] Schwaninger, A. (2009). Why do airport screeners sometimes fail in covert tests? IEEE $43^{\text {rd }}$ International Carnahan Conference on Security Technology, 5-8 October, 2009, Zurich, Switzerland, ICCST 2009, 4145.

[34] Scekic, O. Truong, H-L, Dustdar, S. (2013). Incentives and rewarding in social computing. Communications of the ACM 56(6), 72-82.

[35] Emil, W.O., René, L., and Franziska, H. (2009). A comparison between two leadership models for security checkpoints. 43 $3^{\text {rd }}$ Annual 2009 International Carnahan Conference on Security Technology, 5-8 October, Zurich, Switzerland, ICCST, 59-67. 